Meta

Journal des tradlucteurs

Translators' Journal

\title{
Processus de lexicalisation
}

Modèles psycholinguistiques et leur application à l'étude de

l'aphasie et de la traduction

\section{Guylaine Le Dorze et Jean-Luc Nespoulous}

Volume 29, numéro 1, mars 1984

Cerveau, langage et traduction

URI : https://id.erudit.org/iderudit/002581ar

DOI : https://doi.org/10.7202/002581ar

Aller au sommaire du numéro

Éditeur(s)

Les Presses de l'Université de Montréal

ISSN

0026-0452 (imprimé)

1492-1421 (numérique)

Découvrir la revue

Citer cet article

Le Dorze, G. \& Nespoulous, J.-L. (1984). Processus de lexicalisation : modèles psycholinguistiques et leur application à l'étude de l'aphasie et de la traduction. Meta, 29(1), 68-80. https://doi.org/10.7202/002581ar d'utilisation que vous pouvez consulter en ligne.

https://apropos.erudit.org/fr/usagers/politique-dutilisation/ 


\title{
PROCESSUS DE LEXICALISATION MODĖLES PSYCHOLINGUISTIQUES ET LEUR APPLICATION À L'ÉTUDE DE L'APHASIE ET DE LA TRADUCTION
}

\author{
Guylaine Le Dorze et JeAN-LuC Nespoulous
}

\begin{abstract}
À tout instant, lorsqu'engagés dans des situations de communication, nous faisons appel à notre lexique mental, à l'inventaire des lexèmes connus stockés dans notre cerveau, pour comprendre et reconnaître les mots que nous entendons et lisons, pour décrire maints événements et sentiments, pour exprimer nos pensées, pour nommer personnes, objets et lieux. Instantanément, la plupart du temps, les mots voulus sont produits ou reconnus sans que nous ayons conscience des processus impliqués dans ces tâches.

Comprendre comment nous accédons au lexique mental, afin de caractériser les mécanismes qui gouvernent l'utilisation du langage humain, voilà une des préoccupations de la psycholinguistique. Dans le contexte du présent travail, nous aborderons cette question dans les termes suivants :

a) Comment arrivons-nous à reconnaître un item lexical (lu, entendu, produit) comme étant un mot connu et de ce fait, comment allons-nous le retrouver dans notre stock lexical mental?

b) Comment arrivons-nous à associer à un objet ou à un événement donné l'étiquette lexicale appropriée?

c) Comment s'effectue le processus de recherche lexicale dans le contexte de la production de phrases?

Si l'on admet que le cerveau contient effectivement une représentation de chacun des items lexicaux de la langue que l'on parle, si l'on admet que ces lexèmes constituent des unités psycholinguistiques manipulables - c'est-à-dire accessibles isolément lors de toute entreprise sémiotique (chaque fois qu'un message est transmis d'un individu à un autre) - encore convient-il de savoir sous quelle forme et selon quels principes ils se trouvent organisés dans ce qu'il est convenu d'appeler le lexique mental.

Les " lois et règlements " d'une telle organisation ne sont à l'heure actuelle qu'hypothèses, si grande est la difficulté de leur appréhension. En effet, comment saisir raisonnablement chacun des processus psycholinguistiques à l'oeuvre dans l'opération de " mise en mots " étant donné la grande rapidité à laquelle ils se déroulent (en temps réel) et compte tenu du fait qu'ils échappent quasi totalement à la conscience du sujet parlant ? Pour aussi complexe qu'elle soit, cette tâche constitue le lot quotidien des psycholinguistes. Certains ont abordé le problème par le biais de techniques expérimentales souvent fort ingénieuses. D'autres (Garrett 1980) se sont appuyés sur les erreurs de performance des locuteurs normaux pour étayer leurs théories, tandis que d'autres encore ont utilisé l'approche logico-déductive pour tester leurs hypothèses sur quelques catégories lexico-sémantiques bien définies. Parallèlement, les neurolinguistes, dans le con-
\end{abstract}


texte d'une démarche voisine de celle de Garrett, ont observé les capacités et les erreurs de lexicalisation que font en grand nombre les aphasiques.

Dans le contexte du présent article, nous présenterons plusieurs modèles psycholinguistiques ayant trait (1) à l'accès au lexique, (2) à l'organisation du lexique mental et (3) à la lexicalisation (ou "mise en mots"), autant de thèmes qui nous paraissent susceptibles d'intéresser le traducteur lorsque celui-ci tente d'appréhender les processus mentaux à la base de l'activité traduisante. Les modèles retenus pour cette présentation l'ont été sur la base de leur notoriété. Les controverses qu'ils ont engendrées ne seront pas évoquées. Nous nous contenterons d'atténuer leur dogmatisme apparent par des observations personnelles issues de nos travaux dans le domaine de l'aphasie. Enfin, nous verrons spéculativement ce que de tels modèles peuvent apporter à l'appréhension des mécanismes neuropsycholinguistiques impliqués dans l'opération de traduction.

\section{L'ACCÈS AU LEXIQUE MENTAL ET LE PROCESSUS DE LEXICALISATION}

- Nous présenterons ici, tout d'abord le modèle proposé par Forster (1976). Cette théorie a été élaborée pour rendre compte d'un nombre croissant de résultats issus de travaux utilisant comme paradigme expérimental des "tâches de décision lexicale ". Dans le contexte de telles tâches, un sujet se doit d'indiquer au fur et à mesure que des mots et non-mots (construits selon les règles graphémiques de la langue étudiée) lui sont présentés lesquels appartiennent à la langue et lesquels ne lui appartiennent pas. Les temps de réaction - révélateurs du travail cognitif effectué par le sujet pour résoudre la tâche - sont calculés. Le résultat stable et bien documenté obtenu grâce à ce type d'expérimentation est que la fréquence de l'utilisation du mot est d'une influence certaine sur l'accès lexical. C'est dire que les mots les plus fréquents sont reconnus moins rapidement que les mots plus rares et que les non-mots sont reconnus moins rapidement que tous les mots. Forster a donc conclu de ces observations que le principe organisateur du lexique mental était la fréquence d'utilisation du mot dans la langue.

Examinons le détail des mécanismes et représentations proposés dans le cadre de ce modèle.

\section{FIGURE 1}

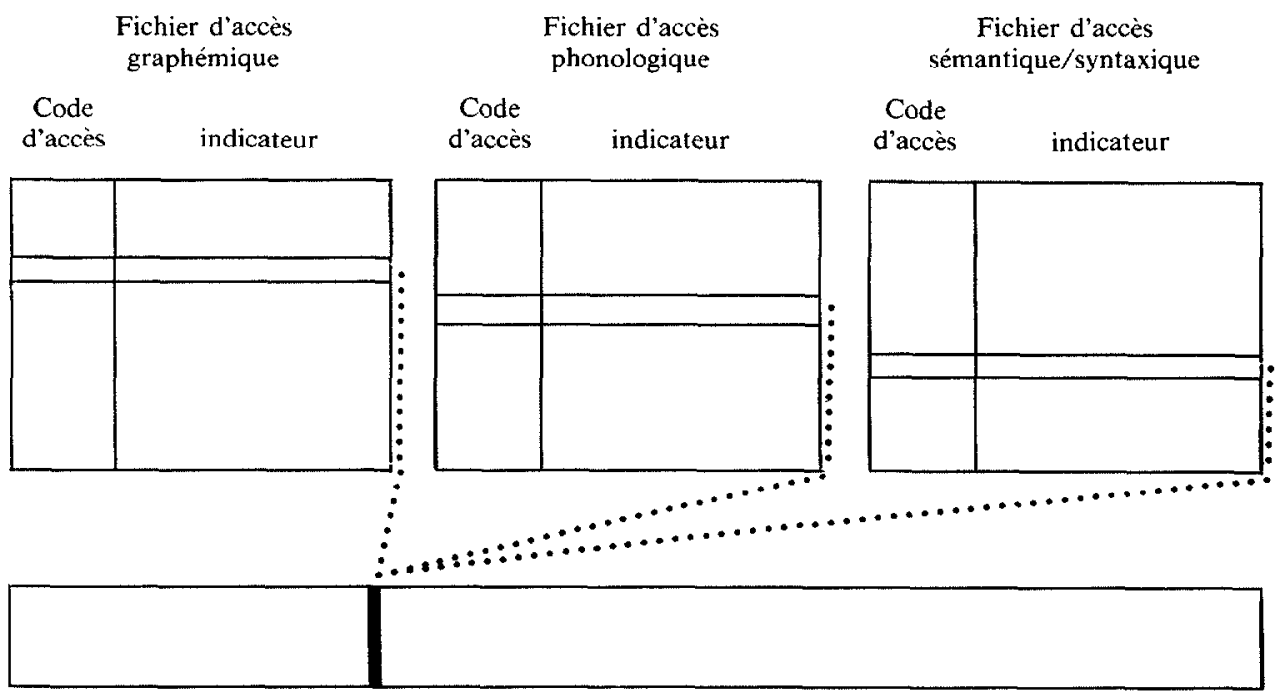

FICHIER CENTRAL OU LEXIQUE MENTAL 
L'accès au lexique se ferait via divers fichiers périphériques représentant les conditions d'utilisation du lexique, soit pour la lecture, la compréhension des messages entendus, et pour la production. Le "fichier lecture" serait organisé selon les propriétés graphémiques des items qu'il contient ; le "fichier compréhension ", selon la structure phonologique-acoustique des items qu'il contient ; et le "fichier production ", selon les propriétés syntaxiques et sémantiques des items lexicaux. Ces fichiers périphériques, indépendants les uns des autres, seraient divisés en groupes d'items de fréquences diverses et contiendraient une description codée des items permettant l'accès au fichier central (ou lexique mental). Avant l'accès au lexique mental, le fichier périphérique examinerait l'item stimulus, le transformerait dans un code reconnu par ce fichier d'accès, ensuite un indicateur retrouverait l'item dans le lexique central. Ainsi l'item stimulus serait reconnu comme mot de la langue, après des temps de latence plus ou moins longs selon le temps de recherche requis, fonction de la fréquence. Un autre résultat clé, obtenu par ce type d'expérimentation, mérite d'être rapporté. C'est ce qui a été appelé l'effet de facilitation sémantique selon lequel on observe une diminution du temps de réaction pour la reconnaissance d'un stimulus lorsque celui-ci a été précédé par la présentation d'un item apparenté sur le plan sémantique. L'explication fournie par Forster veut que l'accès au lexique central ne repasse pas par le fichier périphérique pour la reconnaissance du deuxième stimulus; de ce fait, la fréquence n'est pas toujours le seul facteur qui influence l'accès au lexique, d'autres facteurs, probablement d'ordre sémantique, ont sans doute un rôle à jouer dans cette activité. Malheureusement, Forster élabore peu sur ce dernier point $:$ il ne donne pas plus d'explications que celles que nous venons de rapporter. De plus, même s'il reconnaît que les aspects sémantiques et syntaxiques du lexique sont importants dans la production, il ne va pas plus loin que de proposer l'existence d'un tel fichier, sans indiquer comment il fonctionnerait lors de la production lexicale.

À la lumière de tels effets de facilitation sémantique, on serait tenté d'avancer l'hypothèse selon laquelle le facteur de parenté sémantique serait plus important que celui de la fréquence dans l'organisation du lexique. Tout au plus, pourrions-nous admettre, avec Forster, que la fréquence du mot influence le temps de reconnaissance pour proposer ensuite, contrairement à ce que postule cet auteur, que d'autres facteurs - probablement sémantiques - jouent un rôle dans l'organisation du lexique. Nous ferions ainsi une distinction entre les processus d'accès proprement dits et les facteurs qui l'influencent, d'une part, et la représentation lexicale centrale et ses principes organisationnels, d'autre part, distinction qui n'apparaît pas clairement dans les travaux de Forster.

Finalement, Forster ayant surtout travaillé sur les processus de reconnaissance des mots, son modèle s'avère peu utile pour comprendre le processus de lexicalisation. On pourrait imaginer tout au plus que la lexicalisation est elle aussi influencée par la fréquence de l'item lexical à produire : que lorsqu'un mot de vocabulaire rarement utilisé doit être produit, ce processus est relativement lent en comparaison avec des items lexicaux fréquents. Les études sur les pauses dans le discours conversationnel tendent à appuyer une telle hypothèse (Goldman-Eisler 1968). Ceci étant, nous ne saurions aller plus loin dans la description des processus de lexicalisation sans avoir recours à d'autres modèles que celui de Forster.

- Miller et Johnson-Laird (1976) proposent justement un tout autre point de vue sur cette question, en s'attardant surtout sur les processus qui permettent l'association d'étiquettes lexicales aux objets et événements dont est témoin l'être humain. Dans leur ouvrage, désormais classique, ils réenvisagent complètement la relation entre per- 
ception et langage pour dépasser la notion simpliste issue des associationnistes du début du siècle, selon laquelle le nom (lexème) d'un objet est appris à force de multiples répétitions du nom en présence de l'objet. Leur travail a donné naissance à de nouvelles hypothèses qui pourraient servir de base à des travaux sur la relation perception-langage. En s'appuyant sur de nombreux travaux déjà entrepris sur ce sujet, ils ont vérifié leurs hypothèses en examinant la plausibilité de celles-ci sur des catégories lexico-sémantiques bien définies (couleurs, relations temporelles, verbes de mouvement, par exemple). Ils sont ainsi arrivés à proposer une théorie explicative de l'organisation du lexique mental.

Nous donnerons un bref aperçu de quelques-unes de leurs propositions, en particulier celles qui nous permettent de comprendre comment le lexique est lié à la perception.

\section{FIGURE 2}

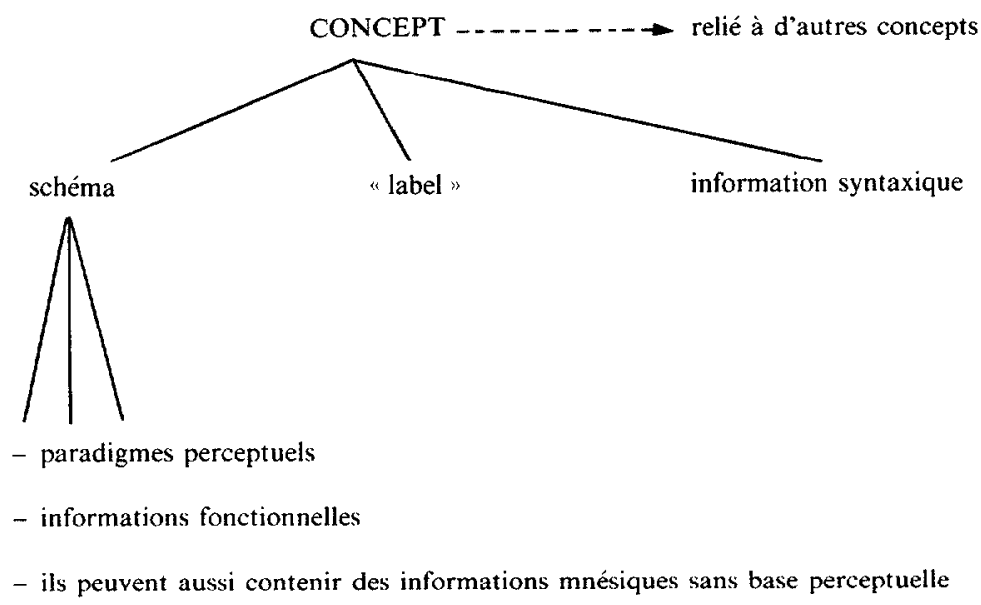

Premièrement, ces auteurs conçoivent la perception en tant qu'activité structurée dont le résultat réside dans l'extraction de caractéristiques distinctives d'un objet (forme, couleur, dimension...) qui s'organiseront en un paradigme perceptuel. Grâce à cette organisation, la vérification est possible : l'objet $\mathrm{x}$ (ayant les caractéristiques $\mathrm{y}, \mathrm{z}$, w) correspond ou pas au paradigme perceptuel de tel ou tel concept.

La perception est donc une activité de conceptualisation parce qu'elle fait référence à des "schémas » qui sont une représentation des informations perceptuelles pertinentes pour un concept. Ces schémas contiennent, en plus des caractéristiques perceptuelles, les informations fonctionnelles et mnésiques sans base perceptuelle se rapportant à l'objet (par exemple, pour l'objet "chaise", le fait qu'elles servent pour s'asseoir, pour travailler à une table..., sont des types d'information qui pourraient être représentés dans les schémas). Le niveau conceptuel agit comme lien entre les schémas perceptuels d'une part et les lexèmes d'autre part. Les lexèmes et les concepts sont englobés par les chanps sémantiques. Le champ conceptuel est constitué des schémas, des "labels ", qui sont le nom du paradigme perceptuel (il ne s'agit pas du lexème à propre- 
ment parler, mais de l'étiquette qui permet l'identification du concept), et de l'information syntaxique telle que la catégorie grammaticale. D'un autre côté, les lexèmes ou les champs lexicaux eux, contiennent le sens linguistique dérivé à partir des relations que les lexèmes entretiennent les uns avec les autres via des routines du type : IS A (est un), HAS A (a un), IS IN (est dans)...

On conçoit ainsi que le lexique mental - ou plutôt les champs sémantiques pour respecter la terminologie de Miller et Johnson-Laird - ont deux composantes : l'une lexicale où des relations diverses (d'inclusion de classe, syntagmatiques, paradigmatiques, partie-tout...) interviennent entre les lexèmes, l'autre conceptuelle contenant les divers traits perceptuels, l'information syntaxique minimale, ainsi que toute autre information mnésique (émotive, fonctionnelle...).

Concrètement, un tel système fonctionnerait comme suit : en présence d'un objet $x$, une analyse visuelle perceptuelle en déterminerait les caractéristiques importantes et les attributs fonctionnels, s'il y a lieu. Ces informations seraient comparées aux paradigmes perceptuels possibles jusqu'à ce que certaines vérifications d'ordre perceptuel déterminent qu'il s'agit bien du schéma y, et donc du " label »w. Des routines feraient le lien entre le schéma $y$, le " label » $w$, l'information syntaxique donnant pleinement accès à la représentation conceptuelle et permettant ainsi l'identification de l'objet $x$. Le lexème $z$ serait activé par le biais (encore) de routines. Par la suite, si nécessaire, d'autres lexèmes pourraient être associés au lexème $\mathrm{z}$ par les relations "IS A ", "HAS A ", "IS IN".

Ce bref résumé de l'architecture théorique du modèle de Miller et Johnson-Laird permet de mesurer la complexité des relations entre langage et perception. Mais malgré la plausibilité de la plupart de leurs hypothèses, la vérification expérimentale auprès de personnes normales ou aphasiques n'est pas évidente. Leur approche demeure donc théorique. De plus, si l'on en vient à examiner le contenu des représentations conceptuelles d'événements que traite le cerveau humain dans lesquels il n'y a pas de base perceptuelle — tels certains lexèmes qui ont été appelés " abstraits 》 : amour, haine, etc. —, nous ne savons comment intégrer ces concepts dans l'échafaudage de Miller et JohnsonLaird. Soulignons quand même que tel n'était pas leur but.

Enfin, en ce qui concerne les processus de lexicalisation, ce modèle nous permet d'envisager assez clairement comment procède le locuteur dans une tâche de dénomination d'objets jusqu'au moment où le lexème approprié a été identifié. Par contre, ils ne disent rien de ce qui se passe dans les étapes subséquentes. Demeurent donc entières les questions suivantes : les lexèmes sont-ils représentés sous leurs aspects formels (phonologiques) dans les champs sémantiques ou seulement sous leur aspect sémantique? Quelles sont les étapes subséquentes du processus de production? Y a-t-il des mécanismes de feed-back qui permettent de vérifier si l'item lexical produit (verbalisé) correspond au lexème choisi ou encore au schéma perceptuel spécifié au départ? De plus, lorsque la verbalisation n'est pas suscitée par une expérience perceptuelle, comment a-t-on accès aux champs sémantiques? Autre question que les auteurs ne se sont pas posés.

- Les questions que nous venons d'évoquer nous mènent à l'examen du dernier modèle que nous souhaitons examiner ici : celui de Garrett. Garrett s'est particulièrement intéressé aux processus de mise en place et de production de phrases ainsi qu'aux mécanismes de la lexicalisation. Son modèle a été élaboré à partir d'observations très différentes de celles qui ont servi de base aux modèles que nous venons d'examiner, dans la mesure où il repose sur une analyse minutieuse des différents types d'erreurs de production observées chez des locuteurs adultes normaux de langue anglaise ${ }^{1}$. Garrett soutient qu'une étude des erreurs de performance permet d'extraire les règles et les procé-

1. Nous n'entrerons pas dans le détail de l'analyse des erreurs qui a permis la mise en place du modèle de 
dures de fonctionnement de la production linguistique qui autrement ne sont pas "visibles". Les erreurs ne sont pas émises au hasard; elles sont d'un nombre relativement restreint, ce qui a permis à Garrett de reconstituer les règles qui gouvernent les processus syntaxiques, lexicaux et phonologiques intervenant entre la "pensée " initiale et la parole articulée finale.. (Voir figure 3).

FIGURE 3

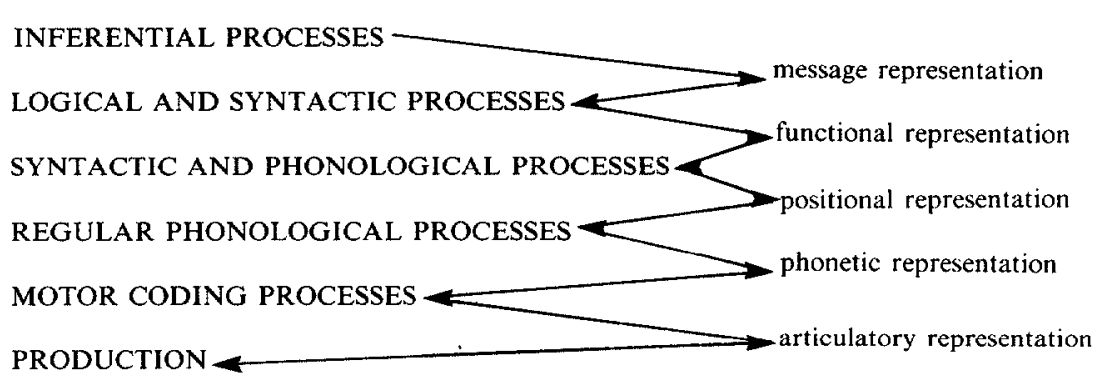

Garrett propose comme étape initiale les "inferential processes" 2 qui permettent à l'individu de tirer de son expérience générale, affective et perceptuelle et des intentions qu'il veut transmettre, une première représentation du message. Celle-ci comprend donc des informations linguistiques aussi bien que non linguistiques et celles-ci sont structurées conceptuellement. Les processus logiques et syntaxiques permettent la sélection d'items lexicaux à partir des aspects sémantiques qui devront être transmis, la spécification des structures fonctionnelles et la mise en place des lexèmes dans celles-ci. Ainsi, la représentation fonctionnelle est déterminée. Sa traduction en représentation positionnelle permet le passage de la forme logique du message à une formulation ordonnée en vue de la production finale. Les formes lexicales sont retrouvées encore via le lexique, mais il s'agit bien maintenant de la spécification des aspects formels des lexèmes. Puis les items grammaticaux sont choisis et placés dans l'ordre dans lequel ils apparaîtront dans la structure de surface. Les processus phonologiques permettent une représentation phonétique de la structure, puis les processus moteurs amènent la représentation articulatoire et la production finale.

Examinons de plus près ce que Garrett dit du processus de lexicalisation. Garrett propose une dualité des processus d'accès lexical : sélection lexicale initiale axée sur les aspects sémantiques, puis re-sélection lexicale, cette fois-ci reposant sur les aspects for-

production. Le lecteur intéressé pourra consulter Garrett $(1980,1984)$ directement.

2. Une traduction adéquate n'ayant pu être trouvée, nous gardons le terme anglais original. 
mels du lexème. La deuxième sélection lexicale se ferait grâce à un lien qui permettrait l'identification de la forme : ce lien contiendrait déjà certaines informations, telles que le premier phonème, le nombre de syllabes, la place de l'accent. Cette dualité serait fondée d'après Garrett puisqu'il n'existe que deux types d'erreurs lexicales chez les locuteurs normaux et chez les aphasiques : (a) erreurs lexicales sémantiques où il y a parenté sémantique entre la forme produite et celle qui aurait dû être produite et (b) erreurs lexicales formelles où des ressemblances formelles existent entre la cible prévue et l'item lexical produit. Donc, nous pourrions conclure que ces types d'organisation sont distincts et qu'il y aurait deux lexiques, l'un à base sémantique et l'autre à base formelle.

On voudrait sans doute, après ces considérations, que Garrett élabore davantage sur ce qu'il entend par la "sélection lexicale "à partir de "spécifications sémantiques", qu'il définisse ce que sont les spécifications sémantiques et qu'il prenne davantage position sur la nature des processus de recherche lexicale proprement dits. Malheureusement, il demeure laconique sur ces derniers points. En fait, il faut aussi comprendre que la théorie sémantique ne permet pas encore de saisir ce que sont les représentations sémantiques dans le système linguistique, ni de comprendre quelles relations il y a entre la sémantique et le lexique. Donc, les psycholinguistiques qui cherchent à savoir comment les aspects sémantiques de la langue s'actualisent dans la production verbale sont incapables d'aller plus loin que la simple constatation selon laquelle l'accès lexical doit comporter une dimension sémantique quelconque.

En terminant cette section sur les processus de lexicalisation, soulignons le fait que le modèle de Garrett a le grand avantage d'envisager comment la lexicalisation s'inscrit dans l'ensemble des processus nécessaires à la production de phrases. Miller et Jonhson-Laird indiquent seulement de quelle manière les relations entre les activités de perception et les représentations lexicales peuvent être envisagées. Forster de son côté souligne le rôle de la fréquence d'utilisation des mots dans l'accès lexical.

En dépit de leur caractère lacunaire, nous verrons dans la prochaine section comment les modèles évoqués précédemment, aussi hypothétiques soient-ils, s'avèrent utiles pour rendre compte des déficits de lexicalisation observés chez l'aphasique.

\section{NEUROLINGUISTIQUE - DÉFICITS DE LEXICALISATION}

Une perturbation présente chez tous les sujets atteints d'aphasie est ce qu'on a appelé classiquement le " manque du mot ". Il s'agit d'une difficulté, voire d'une impossibilité, à produire les vocables spécifiques (mots) de la classe lexicale ouverte (noms, verbes, adjectifs) dans un contexte de discours continu, narratif ou d'épreuves contraignantes particulières (la dénomination d'images, par exemple). Souvent considéré, en clinique, comme un déficit unique, ce trouble de la "lexicalisation " revêt des formes diverses et variées.

Dans la présente section (1) nous présenterons différentes manifestations du manque du mot chez l'aphasique, (2) nous examinerons comment la littérature neurolinguistique tente d'expliquer ce déficit et enfin (3) nous verrons en quoi les modèles précédemment évoqués nous permettent de mieux analyser et de mieux comprendre les erreurs de lexicalisation. De très nombreux travaux ont été effectués, surtout dans la dernière décennie, sur les troubles lexico-sémantiques dans l'aphasie (voir Lesser 1978 ; Buckingham 1981 ; Caramazza et Berndt 1978 ; Le Dorze 1982). Nous ne pouvons ici qu'en donner un bref aperçu.

Plusieurs chercheurs ont tenté de savoir quels étaient les facteurs qui influençaient le manque du mot : type de mot (longueur, fréquence...), contexte de présentation de l'objet à dénommer (isolé, avec d'autres items de la même catégorie ; présentation par diverses modalités sensorielles...). Comme résultat important, on a essentiellement re- 
marqué - comme déjà indiqué - que les items lexicaux plus fréquents dans la langue étaient plus rapidement évoqués que les mots plus rares et qu'ils donnaient lieu à moins d'erreurs dans une tâche de dénomination (Rochford \& Williams 1962, 1963, 1965). Cette observation concorde bien avec ce que Forster fournit comme résultats chez les sujets normaux. Ainsi l'on constate que la fréquence des mots influence la rapidité et la précision avec laquelle les aphasiques effectuent l'accès lexical dans une tâche de dénomination.

D'autres études ont évalué le savoir lexical des sujets aphasiques dans le but de voir s'ils avaient des troubles au niveau de la représentation des informations sémantiques. De nombreux chercheurs suggèrent que les aphasiques et, en particulier, les aphasiques de Wernicke (présentant des lésions postérieures de l'hémisphère gauche) ont des troubles au niveau sémantique : ils ne peuvent classer des items lexicaux selon un superordonné (Lhermitte, Dérouesné \& Lecours 1971; Grober, Perecman, Keller \& Brown 1980), ne peuvent apparier des mots selon leurs traits sémantiques communs (Zurif, Caramazza, Myerson \& Galvin 1974 ; Shanon 1978) ; ils ont aussi des difficultés à reconnaître des mots en relation asociative avec une cible (Goodglass \& Baker 1976). Cependant, ces travaux ne parviennent pas à mettre en relation les troubles "sémantiques " $e t$ les troubles de production lexicale. Il n'est donc pas possible de savoir si les troubles au niveau de la représentation sémantique des mots perturbent les processus de lexicalisation proprement dits.

Ces observations sur les troubles qu'ont les aphasiques dans la manipulation de lexèmes ordonnés selon divers types de relations/associations, viennent appuyer les hypothèses de Miller et Johnson-Laird et démontrent qu'il est plausible de postuler que ce genre de représentation lexicale existe dans le système linguistique ; mais, encore une fois, les implications concernant la production lexicale proprement dite restent obscures.

Quant aux travaux reposant sur la dénomination d'images d'objets, ils n'ont en général fait l'objet que d'une analyse parcellaire, au sein de laquelle, par exemple, seules les premières réponses des sujets ont été analysées (Dubois, Hécaen, Cunin, Daumas, Lerville-Anger \& Marcie 1970 ; Goldblum 1972 ; Marshall 1970). Ces études ont ainsi négligé les tentatives multiples que font souvent les aphasiques pour produire le mot cible attendu, tentatives qui d'ailleurs permettent parfois au patient d'atteindre son but. Ces erreurs et séquences d'approximations lexicales semblent un matériau de choix pour étudier les processus d'encodage lexical et les stratégies développées par le patient pour pallier son déficit.

Examinons quelques exemples de réponses obtenues en dénomination.

paraphasies verbales sémantiques

cerise $\rightarrow$ raisin

brocoli $\rightarrow$ épinard

volcan $\rightarrow$ cratère produit.

On remarque une ressemblance sémantique entre le mot cible et celui qui a été

paraphasies verbales formelles

balai $\rightarrow$ ballon

sifflet $\rightarrow$ filet

rateau $\rightarrow$ gateau 
La ressemblance entre la cible et le mot produit est de nature formelle.

périphrases

perdrix $\rightarrow$ c'est un genre d'oiseau

palmier $\rightarrow$ c'est un arbre qu'on trouve dans le sud

castor $\rightarrow$ il mange du bois, lui... c'est l'emblème du Canada...

il travaille beaucoup...

stéthoscope $\rightarrow$ pour écouter le coeur

Une description ou définition plus ou moins complète est produite.

néologismes

brocoli $\rightarrow$ /mãmidify/

pamplemousse $\rightarrow$ /pãpalyr/

fourchette $\rightarrow / \mathrm{kyla} /$

Il s'agit de segments linguistiques qui ne sont pas des mots mais qui sont employés comme tels.

modalisations

trompette $\rightarrow$ flute... je dis jamais, jamais autre chose

tortue $\rightarrow$ une $/ \mathrm{katy} / .$. peut-être... là je suis pas sûr

serpent $\rightarrow$ souris... presque

cigare $\rightarrow$... faut que je sache là

kangourou $\rightarrow$ ah le verreux... je l'ai pas

cuillère $\rightarrow$ c'est donc bien facile ça caline

girafe $\rightarrow$ je l'connais pas

fourmi $\rightarrow$ je sais pas

Les modalisations portent sur l'acte élocutoire ou le contenu de discours et elles permettent d'appréhender la position du locuteur par rapport à ce qu'il est en train d'émettre... ou de ne pas émettre!

$$
\begin{aligned}
& \text { paraphasies phonémiques } \\
& \text { valise } \rightarrow \text { /valiza/ } \\
& \text { dinosaure } \rightarrow \text { /tigonozor/ } \\
& \text { banane } \rightarrow \text { /bagan/ } \\
& \text { volcan } \rightarrow / \text { kal } \tilde{\alpha} /
\end{aligned}
$$

La série constitutive du mot est anormalement énoncée.

$$
\begin{aligned}
& \text { paragnosies } \\
& \text { dentelle } \rightarrow \text { serpent } \\
& \text { dentelle } \rightarrow \text { chenille } \\
& \text { couronne } \rightarrow \text { tabouret }
\end{aligned}
$$

Les mots substitués et substituants ne sont ni en rapport formel ni sémantiques ; plutôt, les objets qu'ils représentent se ressemblent.

Ces erreurs et verbalisation ont été tirées d'un corpus de production obtenue en dénomination d'images. Toutes les erreurs peuvent être classées en diverses catégories 
dont nous avons retenu les plus importantes. Le nombre de types d'erreurs étant fort limité, il est clair que les déficits de lexicalisation ne sont pas dus au hasard ; ils semblent au contraire respecter certaines règles que nous allons tenter d'expliciter.

Les paraphasies sémantiques semblent clairement indiquer, comme Garrett le propose, qu'il y a sélection lexicale à partir des aspects sémantiques. Ces erreurs sont soit le résultat d'un simple mauvais choix du lexème, soit celui d'une perturbation dans la spécification sémantique qui guide le choix du lexème. Ainsi, si l'information sémantique à lexicaliser est imprécise ou fautive, le choix du lexème peut être erroné et entraîner la production d'une paraphasie sémantique. Il est difficile de juger de ceci en l'absence de commentaires accompagnant d'ordinaire la production de la paraphasie verbale sémantique. Si une modalisation suit la production de l'erreur et qu'elle confirme que la production est bien celle que le sujet avait prévue, on pourrait croire que l'erreur s'est produite au niveau de la spécification sémantique. $\mathrm{Si}$, au contraire, la modalisation indique un rejet de la production, une erreur dans le choix du lexème est plus plausible (sans que le niveau des spécifications sémantiques soit atteint).

Les périphrases démontrent, lorsqu'elles sont informatives, que les spéculations sémantiques sont préservées plus ou moins entièrement (elles sont sans doute un palliatif pour suppléer à l'absence du mot).

Les paraphasies verbales formelles, de leur côté, pourraient permettre d'envisager l'existence de processus formels dans la sélection lexicale. Il faut cependant ajouter que ce type de paraphasie est souvent produit par des aphasiques qui font des paraphasies phonémiques en grand nombre (les aphasiques de conduction). Il ne serait alors pas impossible de croire que certaines paraphasies phonémiques résultent en la production de mots de la langue (Lecours et Lhermitte 1980) tout à fait fortuitement.

Les néologismes et les paraphasies phonémiques résultent tout probablement d'erreurs dans les processus phonologiques, appuyant ainsi une section du modèle de Garrett (cf. supra).

Quoique les paragnosies soient rares chez les aphasiques, elles impliquent que les processus perceptuels font partie de la lexicalisation dans une tâche de dénomination sur images ; une difficulté d'analyse des caractéristiques visuelles d'un objet serait donc dans ce cas à l'origine d'une lexicalisation inadéquate.

Ces observations nous permettent d'envisager quels sont les processus impliqués dans la lexicalisation (voir figure 4). Lorsqu'elle s'effectue à partir d'une stimulation perceptuelle précise (visuelle, par exemple), les processus d'analyse visuelle sont impliqués et permette d'identifier l'objet en présence. La sélection lexicale dépend, d'une part, de spécifications sémantiques précises et, d'autre part, de spécifications formelles qui permettent de retrouver la forme lexicale souhaitée. Par la suite, les processus phonologiques et articulatoires mènent à la réalisation du lexème.

Des difficultés dues à l'un ou l'autre de ses niveaux perturbent le processus de lexicalisation que nous pouvons décrire de la façon suivante. Une erreur dans l'analyse perceptuelle entraîne une mauvaise identification de l'objet et donc une paragnosie. Une difficulté à l'étape de sélection lexicale à partir des spécifications sémantiques mène à la production de paraphasies verbales sémantiques. Une impossibilité de retrouver la forme lexicale peut se manifester par des périphrases transmettant le contenu sémantique qui ne peut être lexicalisé. Une erreur dans le choix d'une forme lexicale amène une paraphasie verbale formelle. Des perturbations des processus phonologiques de mise en place de la forme résulte en des paraphasies phonémiques ou des néologismes. 
Stimulus (déclenche un enchaînement de réponses)

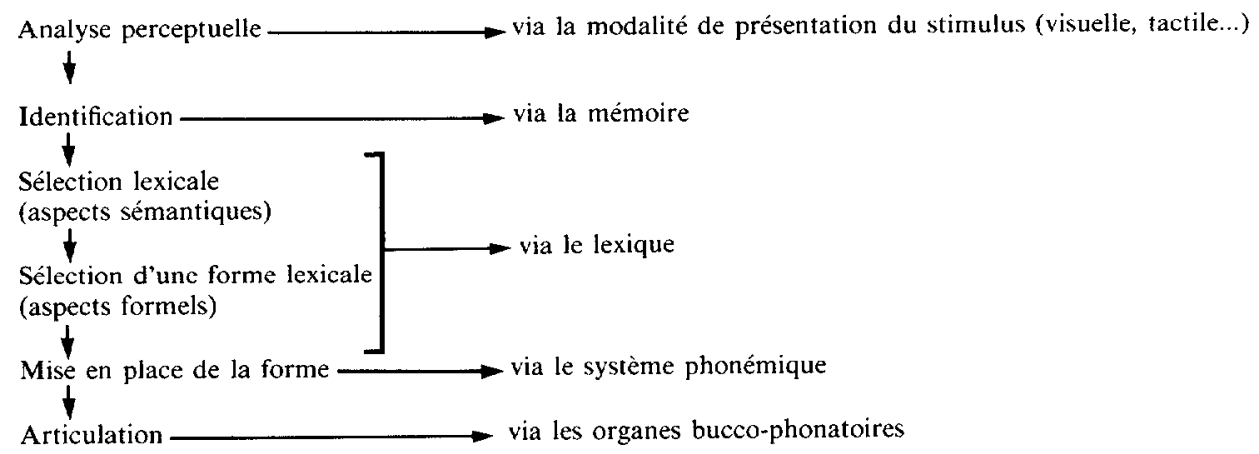

\section{EN GUISE DE CONCLUSION...}

\section{Lexicalisation et traduction}

Bien que les modèles d'accès lexical dont nous avons parlé n'aient pas du tout été conçus pour aborder les mécanismes à l'oeuvre dans l'opération de traduction, nous allons tenter de voir comment ils pourraient aider à atteindre un tel objectif. Postulons dès le départ, que la personne qui traduit possède deux langues et deux lexiques représentés de façon relativement autonome dans le cerveau.

La vision qu'a Forster de l'accès lexical, on le sait (cf. supra, ne fait probablement pas intervenir l'aspect sémantique des unités lexicales : l'accès se fait grâce à un processus relativement automatique fonctionnant selon la fréquence de l'item lexical à traiter. Nous pouvons dès lors imaginer que la traduction est elle aussi tributaire de la fréquence des items lexicaux dans chacune des langues impliquées (d'où l'extrême difficulté de la tâche de traduction dans des domaines peu familiers du traducteur). Toujours en suivant Forster, nous pourrions proposer l'existence d'un fichier d'accès «traduction » au sein duquel un indicateur identifierait le lexème équivalent dans la langue à traduire. Il ne serait dès lors pas nécessaire de passer par le lexique central de la langue cible pour accéder au lexème adéquat. Ce type de stratégie de traduction " quasi automatique » pourrait effectivement rendre compte des processus employés pour repérer des items lexicaux très fréquemment utilisés dans le processus de traduction.

Les propositions de Miller et Johnson-Laird nous conduisent à nous demander, en considérant les processus de traduction, s'il y a représentation sémantique (les champs sémantiques) pour chacune des langues, ou encore si les champs sémantiques sont uniques, comprenant un seul niveau de représentation conceptuel auquel sont rattachés deux lexiques $\left(\mathrm{L}_{1}\right.$ et $\left.\mathrm{L}_{2}\right)$. Nous ne pouvons répondre à cette question avec les données que nous avons en main. Cependant, puisqu'il est rare que deux langues traitent de façon identique les données de l'expérience humaine, il est peut-être plus plausible de supposer que les représentations sémantiques de deux langues sont distinctes. Ainsi le pro- 
cessus de traduction de $L_{1}$ à $L_{2}$ supposerait une procédure qui permettrait d'accéder aux champs sémantiques de $L_{2}$ à partir de $L_{1}$. Le lexème $L_{2}$ adéquat serait choisi en fonction des schémas perceptuels incorporés dans la représentation conceptuelle de $\mathrm{L}_{2}$. Cette vision des processus de traduction rendrait compte des situations dans lesquelles le traducteur n'aurait pas de lexème équivalent immédiatement disponible, ce qui l'obligerait à "remonter" au niveau des représentations sémantiques avant de choisir au bout du compte le lexème le plus adéquat pour transmettre le contenu sémantique du mot de $\mathbf{L}_{1}$ à traduire.

En dernier lieu, le modèle de Garrett permet aussi d'avancer quelques hypothèses sur la nature des processus de traduction. Garrett prétend en effet que la lexicalisation, dans le contexte de la production de phrases, passe par la représentation du message, et qu'elle est double, sémantique d'abord, puis formelle. En utilisant ce modèle, nous pourrions supposer que la traduction d'une phrase nécessite une analyse de son contenu, laquelle oblige le traducteur à remonter très en amont dans la chaîne de production, jusqu'au niveau de la représentation du message. À partir de celle-ci, les doubles (sémantiques et formels) processus de lexicalisation seraient mis en place pour obtenir une phrase équivalente dans $\mathrm{L}_{2}$. Cette proposition implique que la traduction se ferait très " haut », à un niveau conceptuel, et qu'elle dépendrait peu d'équivalences directes entre lexèmes (plus en " aval »). On pourrait aussi imaginer différentes stratégies - plutôt en amont ou plutôt en aval - en fonction de la compétence professionnelle et de l'habileté du traducteur... on pourrait alors parler de "stratégies de traduction ", variables d'un traducteur à un autre, plutôt que de processus stables et invariables chez tous les praticiens de cet art.

En termes psycholinguistiques, la question centrale - sans réponse catégorique, nous semble-t-il — demeure la suivante : les processus de lexicalisation à l'oeuvre dans l'opération de traduction impliquent-ils le recours aux niveaux de représentation sémantiques et/ou formels ? Même s'il nous est impossible de répondre à une telle question, les différentes propositions que nous avons examinées suggèrent différentes stratégies de traduction qui, encore une fois, ne sont pas nécessairement mutuellement exclusives. S'il s'agit de retrouver dans le lexique $L_{2}$ un terme de la langue $L_{1}$ qui est très fréquemment traduit par tel ou tel traducteur, ceci se fait sans doute directement, sans aucune participation du niveau de la représentation sémantique en $L_{2}$. Par contre, si le registre "automatique" ne contient pas l'item lexical à traduire, il est raisonnable de postuler que le niveau sémantique est lui aussi impliqué. Finalement, dans le cas de la traduction fidèle et approfondie de tel ou tel corpus, on conçoit aisément que les processus mis en oeuvre impliquent le recours au niveau de la représentation du message, afin que les intentions de l'auteur (en $\mathrm{L}_{1}$ ) soient bien rendues $\left(e n \mathrm{~L}_{2}\right.$ ). La première stratégie s'avérerait particulièrement utile lors de tâches de traduction en temps réel - chez l'interprète - la deuxième stratégie - plus coûteuse à bien des égards - étant plus spécifiquement réservée aux tâches de " traduction différée » comme la traduction de textes écrits.

En résumé, et même si ces dernières lignes se veulent résolument hypothétiques, il y a fort à parier que, pas plus que d'autres comportements humains, la traduction ne repose pas sur des processus invariables et stéréotypés. Si certains processus de ce type existent sûrement, ils sont très certainement accompagnés de stratégies éminemment variables d'un traducteur à un autre en fonction de multiples paramètres... d'où la difficulté de leur appréhension.

\section{RÉFÉRENCES}

BUCKINGHAM, H. (1981) : "Lexical and Semantic Aspects of Aphasia", in M.T. SARNO, Acquired Aphasia, New York, Academic Press. 
CARAMAZZA, A \& R.S. BERNDT (1978) : "Semantic and Syntacic Processes in Aphasia : A review of the Litterature", Psychological Bulletin, 85, pp. 898-918.

DUBOIS, J., H. HÉCAEN, S. CUNIN, M. DAUMAS, B. LERVILlE-ANGER \& P. MARCIE (1970) "Analyse linguistique d'énoncés d'aphasiques sensoriels", Journal de psychologie normale et pathologique, 2, pp. 185-206.

FORSTER, K.I. (1976) : "Accessing the Mental Lexicon", in R.J. WALES \& E. WALKER (Edits), New Approaches to Language Mechanisms, Amsterdam, North Holland Publishing.

GARRETT, M.F. (1980) : "Levels of Processing in Sentence Production", in B. BUTTER WORTH (Edit.), Language Production, Vol. 1, London, Academic Press.

GARRETT, M.F. (1984) : "The Organization of Processing Structure for Language Production : Applications to Aphasic Speech", in D. CAPLAN, A.R. LECOURS \& A. SMITH (Edits), Biological Perspectives on Language, Cambridge, Mass., The MIT Press.

GOLDMAN-EISLER, F. (1968) : Psycholinguistics ; Experiments in Spontaneous Speech, London and New York, Academic Press.

GOODGLASS, H. \& E. BAKER (1976) : "Semantic Field, Naming and Auditory Comprehension in Aphasia", Brain and Language, 3, pp. 359-374.

GOLDBLUM, M.C. (1972) : "Analyse des réponses de dénomination chez les aphasiques ", Langages : neurolinguistique et neuropsychologie, 25 , pp. 66-75.

GROBER, E., E. PERECMAN, L. KELLAR \& J. BROWN (1980) : "Lexical Knowledge in Anterior and Posterior Aphasics", Brain and Language, 10, pp. 318-330.

LECOURS, A.R. \& F. LHERMITTE (1980) : l'Aphasie, Montréal, Les Presses de l'Université de Montréal.

LE DORZE, G. (1982) : "Le manque du mot et l'organisation lexico-sémantique dans l'aphasie ", revue de littérature, manuscrit non publié, Montréal, Centre de recherche du Centre hospitalier Côte-desNeiges.

LESSER, R. (1978) : Linguistic Investigations of Aphasia, London, Edward Arnold.

LHERMITTE, F., J. DEROUESNÉ \& A.R. LECOURS (1971) : "Contribution à l'étude des troubles sémantiques dans l'aphasie ", Revue neurologique, 125, pp. 81-101.

MARSHALL, R.C. (1976) : "Word Retrieval of Aphasic Adults", Journal of Speech and Hearing Research, 41, pp. 444-451.

MILLER, G.A. \& P.N. JOHNSON-LAIRD (1976) : Language and Perception, Cambridge, Cambridge University Press.

ROCHFORD, G. \& M. WILLIAMS (1962): "Studies in the Development and Breakdown of the Use of Names", Journal of Neurology, Neurosurgery and Psychiatry, 25, pp. 222-227, 228-233.

ROCHFORD, G. \& M. WILLIAMS (1983) : "Studies in the Development and Breakdown of the Use of Names : Recovery from Nominal Dysphasia", Journal of Neurology, Neurosurgery and Psychiatry, 26, pp. 377-381.

ROCHFORD, G. \& M. WILLIAMS (1965): "Studies in the Development and Breakdown in the Use of Names : Effects of Word Frequency", Journal of Neurology, Neurosurgery and Psychiatry, 28, pp. 407-413.

SHANON, B. (1978) : "Classification and Identification in an Aphasic Patient", Brain and Language, 5, pp. 188-194.

ZURIF, E.B., A. CARAMAZZA, R. MYERSON \& J. GALVIN (1974) : "Semantic Representations for Normal and Aphasic Language", Brain and Language, 1, pp. 167-187. 